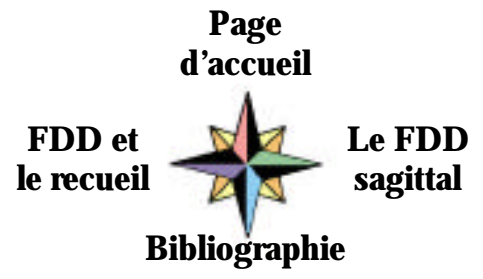

\title{
Les actifs non-agricoles des campagnes françaises : corrélations et autocomélations
}

En raison de la diminution de la part des agriculteurs dans la population active, l'espace nural est de plus en plus largement lieu de résidence et de travail de non-agriculteurs. Nous nous proposons de reprendre et de prolonger ici des études antérieures sur la structure des populations actives non agricoles des campagnes françaises, selon des données actualisées (celles du recensement de 1975), dans des perspectives et avec des techniques un peu différentes1. Malgré des limites évidentes, maintes fois soulignées, l'utilisation à la fois du cadre départemental et des statistiques par catégories socioprofessionnelles nous a paru assez riche d'enseignements pour pouvoir servir de base de recherche.

Nous avons choisi de mettre complètement entre parenthèses la part des agriculteurs, en opérant sur 13 catégories socioprofessionnelles non-agnicoles (voir liste au Tableau 1) et en calculant des pourcentages en fonction du total de ces 13 CSP. En effet, si l'on prend comme référence la population active totale, la variation de la part des agriculteurs est prise en compte "en négatif ", et elle pèse assez sur les résultats pour que les structures de la population non-agricole restent cachées ou, tout au moins, se dégagent moins bien ; de plus, les comparaisons nécessaires avec les populations urbaines sont plus difficiles. L'élimination des agriculteurs pourra évidemment choquer ou étonner, mais elle est nécessaire si l'on veut mettre en évidence des organisations significatives.

Tableau 1 : Codage des variables utilisées

\begin{tabular}{|c|c|c|c|}
\hline & $\begin{array}{l}\text { Ruraux recensés } \\
\text { au lieu de } \\
\text { résidence }\end{array}$ & $\begin{array}{l}\text { Ruraux recensés } \\
\text { au lieu de } \\
\text { travail }\end{array}$ & $\begin{array}{l}\text { Urbains recensés } \\
\text { au lieu de } \\
\text { travail }\end{array}$ \\
\hline Industriels & RRIN & RTIN & UTIN \\
\hline Petits commençants & RRPC & RTPC & UTPC \\
\hline Gros commerçants & RRCC & RTGC & UTGC \\
\hline Professions libérales & RRPL & RTPL. & UTPL \\
\hline Cadres supénieurs & RRCS & RTCS & UTCS \\
\hline Cadres moyens & RRCM & RTCM & UTCM \\
\hline Employés de bureau & RREB & RTEB & UTEB \\
\hline Employés de commerce & RREC & RTEC & UTEC \\
\hline Contremaîtres & RRCO & RTCO & UTCO \\
\hline Ouvriers professionnels & RROP & RTOP & UTOP \\
\hline Ouvriers spécialisés & RROS & RTOS & UTOS \\
\hline Manœuvres & RRMA & RTMA & UTMA \\
\hline Artisans & RRAR & RTAR & UTAR \\
\hline
\end{tabular}

Toutes les valeurs sont en \% par rapport aux totaux des 13 CSP, dans chaque ensemble spatial.

\footnotetext{
${ }^{1}$ Les traitements informatiques ont été effectués à l'Université Paris VII par C. Perez-Diaz et L. Sanders. La technique cartographique utilisée pour la Figure 4 a été suggérée par Mme Pédron.
} 
Les catégories socioprofessionnelles considérées selon leurs effectifs départementaux entretiennent entre elles des rapports sociaux et spatiaux de trois types, qui peuvent être saisis par trois utilisations différentes de calculs de corrélation :

- Les corrélations entre CSP recensées dans un même type d'espace permettent d'identifier celles qui sont simultanément sur ou sous-représentées dans telle ou telle portion du territoire. Les proportions variables des différentes CSP permettent de définir des groupements que l'on peut appeler des " sociétés locales 》 - modulations selon l'espace de la société globale de la France ou, pour reprendre l'expression de A. Reynaud, des classes socio-spatiales. Le Tableau 2 montre que les variations des proportions sont relativement faibles - les coefficients de variation sont très nettement inférieurs à l'unité, et la plupart sont inférieurs à 0,50. Mais des différences qui peuvent apparaître minimes comme caractères d'une société locale (ainsi, le pourcentage des membres des professions libérales varie entre des extrêmes de 0,30 et $1,68 \%$ ), sont toujours l'expression significative d'une fonction régionale, et peuvent donc légitimement retenir l'intérêt, comme résultantes d'un ensemble de caractères de l'espace.

L'étude de ces ensembles de corrélations et des sur et sous-représentations simultanées est facilitée par les techniques d'analyse factorielle.

- Les corrélations entre les effectifs dans les différents types d'espace. Puisque tous les actifs sont recensés d'une part à leur lieu de résidence, d'autre part à leur lieu de travail, l'INSEE distingue en fait quatre espaces : l'espace des communes rurales en tant qu'espace résidentiel et en tant qu'espace de travail, celui des communes urbaines également envisagé des deux points de vue. En fonction de l'accent mis ici sur les campagnes, il est intéressant d'étudier la répartition des CSP recensées au lieu de résidence dans les communes rurales puis de la confronter avec la répartition dans les communes rurales envisagées comme lieu de travail, et dans les communes urbaines. On peut d'ailleurs poser une hypothèse explicative simple : les effectifs résidant dans les communes rurales doivent dépendre d'une part des effectifs travaillant dans les communes rurales, d'autre part de l'activité urbaine, et cela par un double mécanisme : beaucoup de ruraux vont travailler en ville (effet direct); les activités non agricoles dans les villes et dans les campagnes sont liées entre elles et les effectifs résidant dans les campagnes dépendent des effectifs au travail (effet indirect). Cet ensemble de relations est schématisé dans la Figure 1. Les deux effets ont été identifiés par les lettres a (circuit direct) et b (circuit indirect). Ces corrélations ont été mises en évidence encore grâce à des analyses factorielles, mais aussi par l'étude analytique de corrélations choisies, et par des calculs de corrélations partielles.

- Les corrélations entre des «individus spatiaux » proches (ici entre départements voisins). Dans la mesure où il existe des aires, plus étendues que les départements, qui présentent une certaine homogénéité, on doit s'attendre à trouver au moins pour un certain nombre de variables, des comélations positives entre les valeurs pour les départements proches, et négatives pour des départements éloignés. Il s'agit ici de corrélations entre valeurs pour une même variable, donc bien "d'autocorrélations ". En quelque sorte, l'étude de l'autocomélation permet de voir dans quelle mesure « ce qui se ressemble s'assemble "; nous avons utilisé pour cette évaluation l'indice de Geary, qui a été calculé pour 36 variables. 
Tableau 2 : Moyennes et coefficients de variation des variables

\begin{tabular}{llcccccc}
\hline & & \multicolumn{2}{c}{ Résidents ruraux } & \multicolumn{2}{c}{ Ruraux } & \multicolumn{2}{c}{ Urbains } \\
& & \multicolumn{2}{c}{ RR } & \multicolumn{2}{c}{ RT } \\
& & \multicolumn{2}{c}{ RR travail } & \multicolumn{2}{c}{$\begin{array}{c}\text { Lieu de travail } \\
\text { UT }\end{array}$} \\
\hline Artisans & & moy & CV & moy & CV & moy & CV \\
Industriels & AR & $\mathbf{6 , 0 0}$ & $\mathbf{0 , 3 2}$ & $\mathbf{8 , 6 2}$ & $\mathbf{0 , 3 0}$ & $\mathbf{2 , 8 8}$ & $\mathbf{0 , 3 5}$ \\
Petits commerçants & IN & $\mathbf{0 , 4 7}$ & $\mathbf{0 , 3 0}$ & $\mathbf{0 , 6 8}$ & $\mathbf{0 , 2 7}$ & $\mathbf{0 , 3 7}$ & $\mathbf{0 , 3 7}$ \\
Gros commerçants & PC & $\mathbf{7 , 5 6}$ & $\mathbf{0 , 2 6}$ & $\mathbf{1 0 , 4 1}$ & $\mathbf{0 , 2 4}$ & $\mathbf{5 , 3 3}$ & $\mathbf{0 , 2 5}$ \\
Professions libérales & GC & $\mathbf{1 , 0 2}$ & $\mathbf{0 , 3 3}$ & $\mathbf{1 , 3 1}$ & $\mathbf{0 , 3 4}$ & $\mathbf{1 , 2 0}$ & $\mathbf{0 , 2 8}$ \\
Cadres supérieurs & PL & $\mathbf{0 , 7 5}$ & $\mathbf{0 , 3 6}$ & $\mathbf{0 , 9 1}$ & $\mathbf{0 , 3 6}$ & $\mathbf{1 , 0 5}$ & $\mathbf{0 , 2 7}$ \\
Cadres moyens & CS & $\mathbf{3 0 5 2}$ & $\mathbf{0 , 4 3}$ & 3,04 & $\mathbf{0 , 6 7}$ & $\mathbf{6 , 2 7}$ & $\mathbf{0 , 2 5}$ \\
Employés de bureau & CM & $\mathbf{1 2 , 3 9}$ & $\mathbf{0 , 1 5}$ & $\mathbf{1 2 , 3 5}$ & $\mathbf{0 , 1 5}$ & $\mathbf{1 5 , 3 6}$ & $\mathbf{0 , 1 1}$ \\
Employés de commerce & EB & $\mathbf{1 2 , 3 7}$ & $\mathbf{0 , 1 4}$ & $\mathbf{9 , 1 9}$ & $\mathbf{0 , 1 8}$ & $\mathbf{1 6 , 8 6}$ & $\mathbf{0 , 1 1}$ \\
Contremaîtres & $\mathbf{2 , 9 6}$ & $\mathbf{0 , 1 5}$ & $\mathbf{2 , 4 8}$ & $\mathbf{0 , 2 1}$ & $\mathbf{4 , 4 0}$ & $\mathbf{0 , 1 5}$ \\
Ouvriers professionnels & OO & $\mathbf{2 , 1 0}$ & $\mathbf{0 , 3 6}$ & $\mathbf{2 , 0 3}$ & $\mathbf{0 , 3 8}$ & $\mathbf{2 , 5 2}$ & $\mathbf{0 , 2 5}$ \\
Ouvriers spécialisés & $\mathbf{1 6 , 8 9}$ & $\mathbf{0 , 1 4}$ & $\mathbf{1 5 , 8 8}$ & $\mathbf{0 , 1 7}$ & $\mathbf{1 6 , 5 1}$ & $\mathbf{0 , 1 4}$ \\
Manœuvres & OS & $\mathbf{2 2 , 2 4}$ & $\mathbf{0 , 2 1}$ & $\mathbf{2 1 , 0 2}$ & $\mathbf{0 , 2 1}$ & $\mathbf{1 8 , 6 8}$ & $\mathbf{0 , 2 3}$ \\
\hline
\end{tabular}

moy : moyenne ; CV : coefficient de variation

(Valeurs calculées pour les départements français, à l'exclusion de Paris, de la petite couronne et de la Corse. Pour cette dernière, les résultats du recensement de 1975 n'ont pas été considérés comme assez fidèles par l'INSEE pour être publiés avec les autres).

Figure 1: Schéma des liaisons logiques

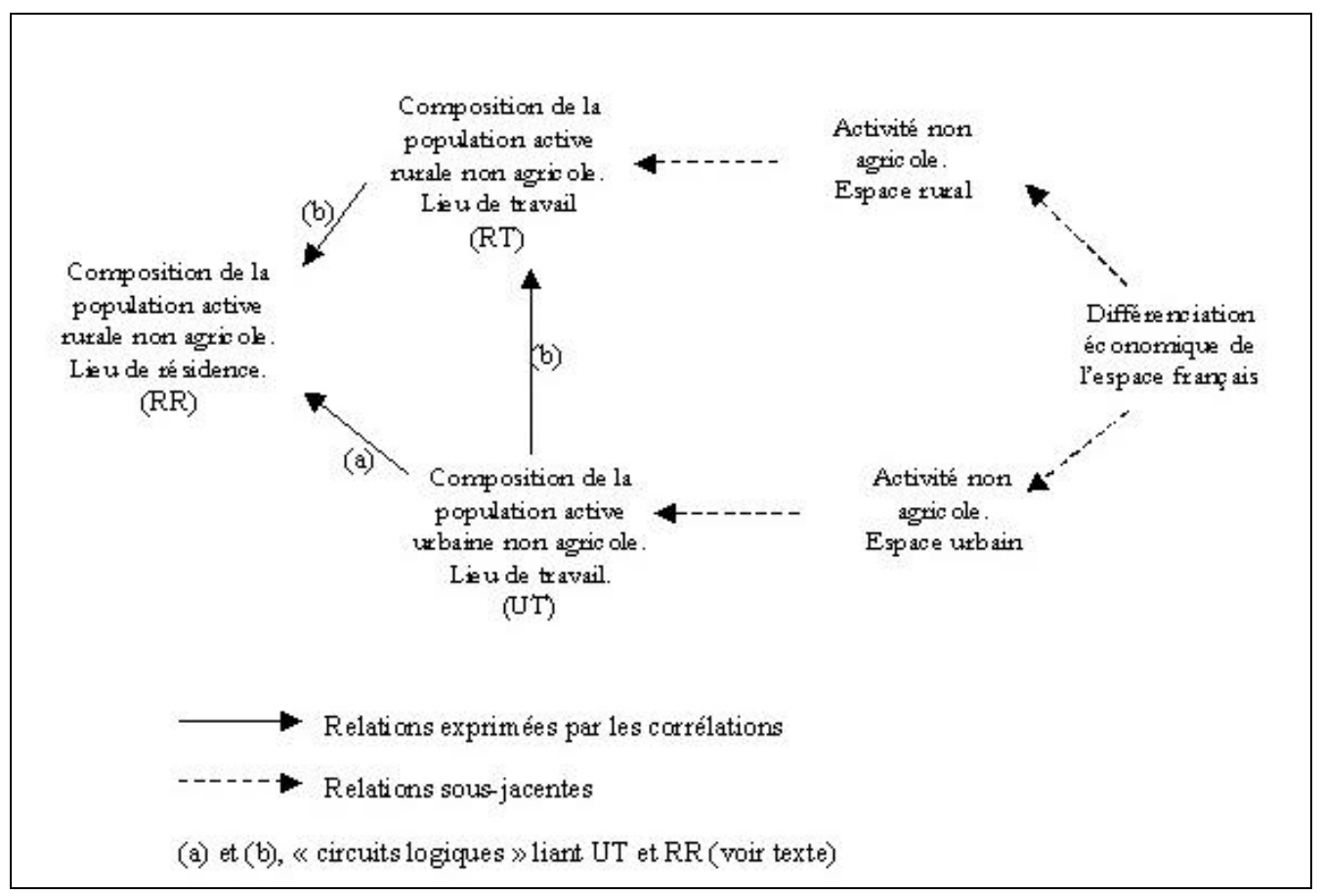

L'existence de rapports entre les caractères des populations des villes et des campagnes d'un même département, entre les populations de départements proches, est évidemment un fait banal. Peu de calculs sont nécessaires pour l'établir, il suffit de regarder des cartes analytiques. Certes. Mais ce qui est souvent moins banal, ce sont les différences entre types d'espaces, entre catégories socioprofessionnelles; peu évidentes a priori, elles ne peuvent être mises en valeur que par des comparaisons, qui, elles mêmes, ne sont possibles que par des mesures assez précises et parfois assez complexes. Ce sont elles, bien souvent, qui permettent de retrouver les spécificités. 


\section{Les classes socio-spatiales rurales}

Une première image de la répartition des groupements de catégories socioprofessionnelles dans l'espace nural est offerte par la carte (cf. Figure 2A) des coordonnées des départements sur les axes 1 et 2 d'une analyse des comespondances portant sur les effectifs des 13 CSP retenues, auxquelles on a ajouté les non-actifs et les étudiants. Il s'agit des actifs recensés au lieu de résidence donc des résidents ruraux non agricoles.

Figure 2A : Actifs 1975 - lieu de résidence (communes rurales)

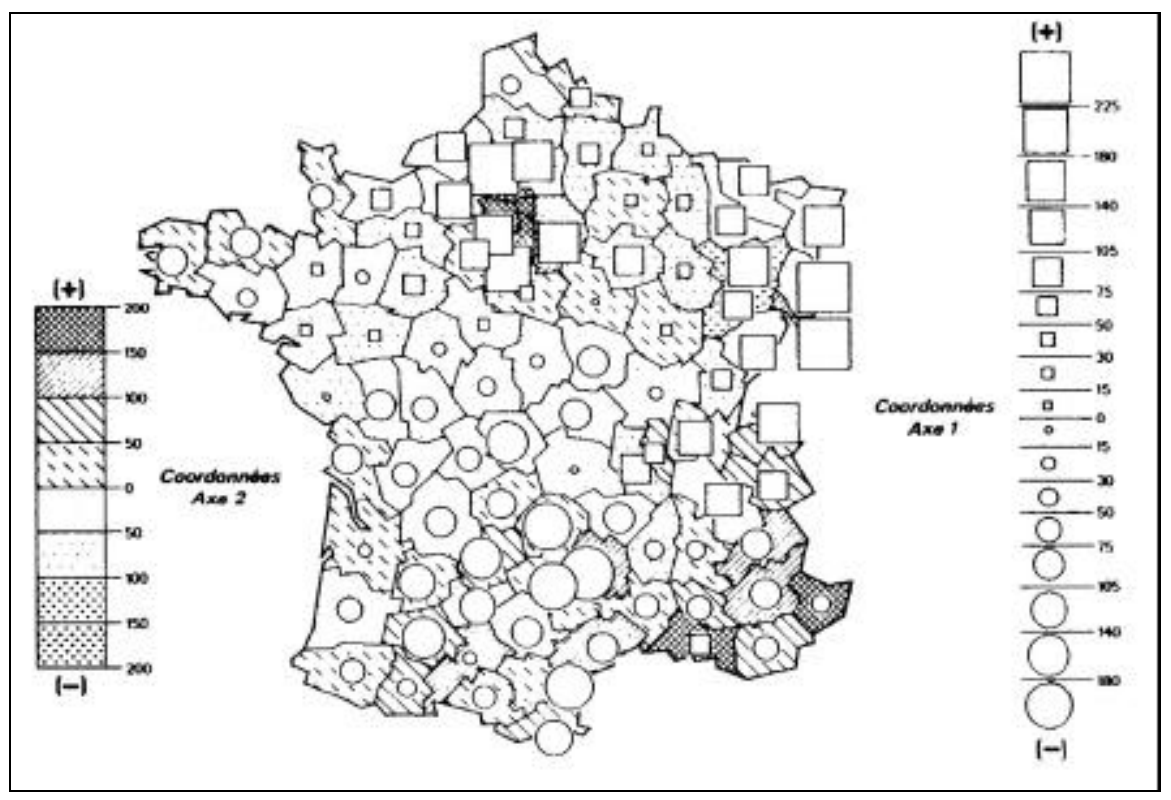

Figure 2B : Actifs 1975 - lieu de résidence (communes urbaines)

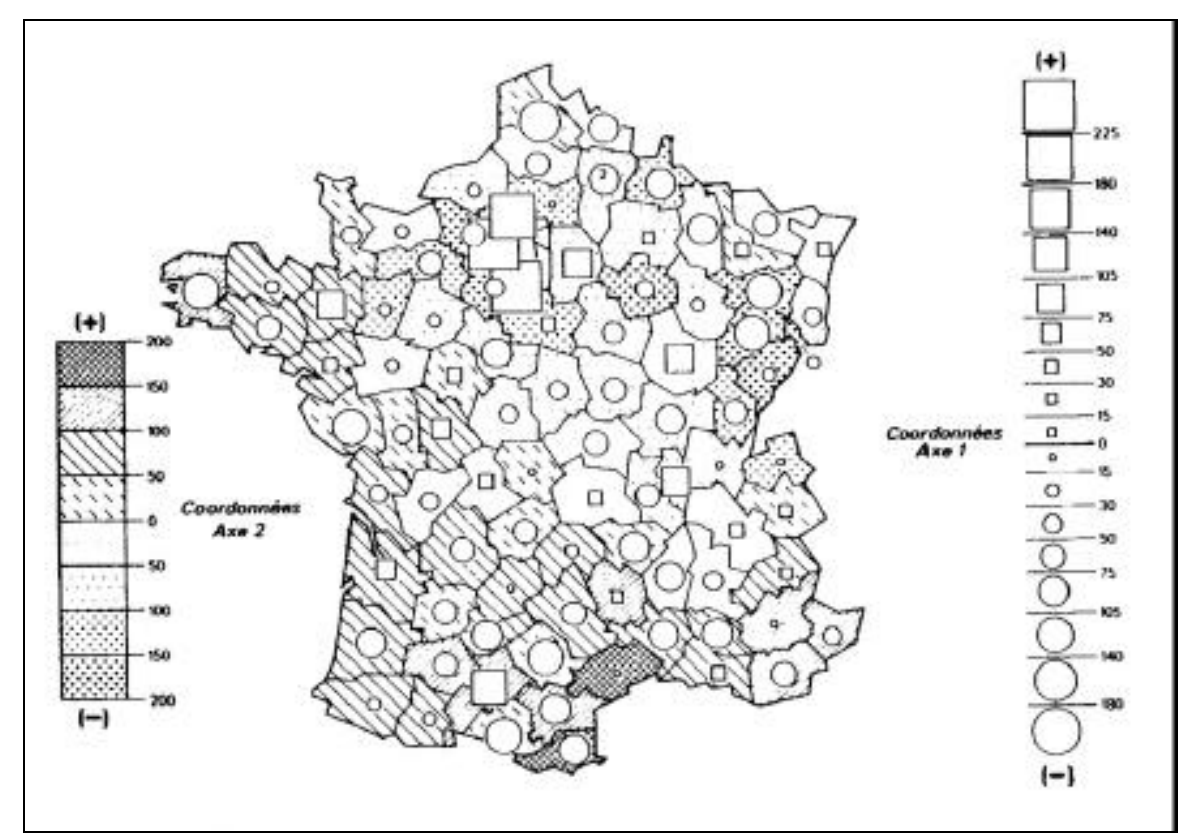


La définition des deux premiers axes, qui prennent en compte près des trois quarts de la variance totale, est assez claire (cf. Figure $2 \mathrm{C}$ ). Le premier oppose nettement les salariés de toutes catégories aux non-salariés, le second, les cadres et les professions libérales d'une part, aux manoeuvres et aux ouvriers spécialisés d'autre part; on serait tenté de définir ce deuxième axe comme révélateur d'une "composante de qualification" du travail : la définition ainsi exprimée est acceptable si l'on ne prend en compte que les extrêmes, mais les variables intermédiaires se rangent plutôt en fonction de "l'image sociale " des CSP dans le système des valeurs courant dans la France contemporaine : souvent moins "qualifiés » que des ouvriers professionnels ou bien des artisans, les employés de bureau sont plus proches qu'eux des cadres et des membres des professions libérales.

Figures 2C et 2D : Ruraux au lieu de résidence

Analyses séparées. Comespondances

Coordonnées des variables sur les axes 1 et 2

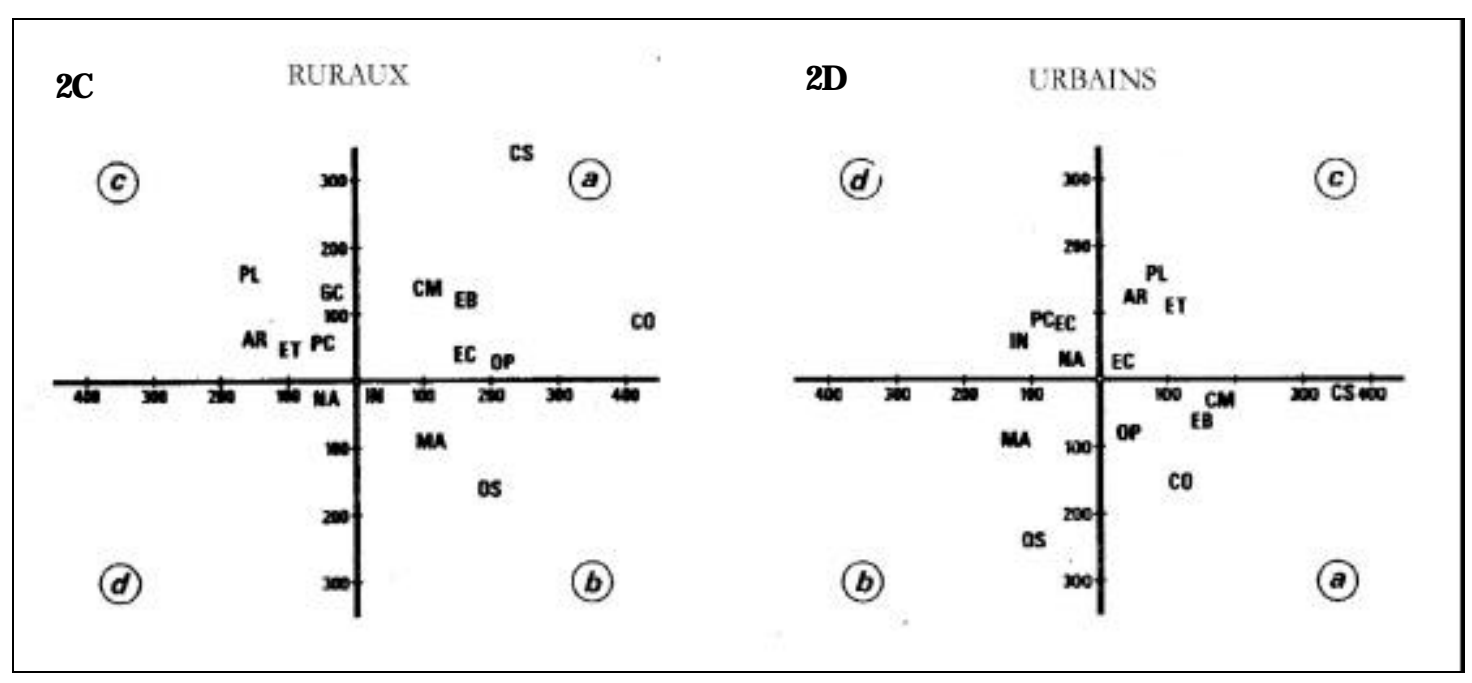

Code des variables : cf. Tableau 1.

Voir texte pour la signification des lettres encerclées.

L'étude de la position des points représentatifs des variables sur le plan définit assez nettement quatre secteurs (identifiés par des lettres sur la Figure 2C).

Secteur a - Des coordonnées positives sur les deux axes correspondent à une surreprésentation relative des actifs qui exercent des fonctions de direction et de conception (cadres supérieurs), qui y collaborent à des titres divers (employés de bureau), ou qui exercent des activités de transformation industrielle demandant une assez forte qualification (contremaîtres et ouvriers professionnels) ; cette combinaison est réalisée dans les départements autour de Paris et de Lyon, et dans les communes rurales des départements où se trouvent des métropoles régionales assez industrialisées, comme Lille, Marseille et, à un moindre degré, Reims ou Dijon (cf. Figure 2A).

Secteur b - Au contraire, des coordonnées négatives sur l'axe 2 et positives sur l'axe 1 reflètent un développement de toutes les activités salariées, mais aussi une nette surreprésentation de ceux qui accomplissent des tâches d'exécution dans le domaine industriel, OS et manouvres, et une sous-représentation des services. Cette combinaison se rencontre dans l'essentiel de la «France industrielle » au nord-est de la toujours 
présente ligne. Le Havre-Genève, mais aussi dans des départements de l'O uest : Ille-etVilaine, Sarthe, Loire-Atlantique, Maine-et-Loire.

Secteur c - Des coordonnées négatives sur l'axe 1 et positives sur l'axe 2 identifient des espaces où les actifs des services, surtout non salariés, sont sur-représentés, alors que les manouvres et $\mathrm{OS}$ sont sous-représentés. Ces caractères s'affirment dans la France méridionale, nettement sur un espace bien continu du Midi méditerranéen, moins nettement et moins régulièrement dans le sud du Massif Central et dans une grande partie de l'Aquitaine.

Secteur d - Des coordonnées négatives sur les deux axes reflètent d'abord un faible développement des activités de décision et de conception, ainsi que du travail industriel qualifié ; une sur-représentation assez peu affirmée des activités de senvices non salariés, des manouvres et des $0 \mathrm{~S}$ vient compléter le tableau. Cette combinaison est réalisée dans le centre et le centre-ouest du pays, quelques régions du sud-est du Massif Central et de l'Aquitaine (Landes, Tarn, T am-et-Garonne).

L'image offerte de la France par la carte est simple, avec des espaces bien cohérents elle reflète des oppositions classiques, bien connues dans leurs grandes lignes : l'opposition de la France "industrielle " et "non industrielle ", la diffusion des tâches d'exécution dans l'Ouest, la concentration des activités de décision dans quelques centres, leur liaison avec les fabrications spécialisées, la concentration relative des services dans le Midi...

\section{Les classes socio-spatiales urbaines et leurs rapports avec les classes socio- spatiales rurales}

Nous les décrirons en utilisant des documents assez différents qui fournissent des indications complémentaires et convengentes :

- les résultats d'une analyse des correspondances portant sur les mêmes variables que celles utilisées ci-dessus, mais pour les CSP recensées au lieu de résidence dans les villes et les communes urbaines (cf. Figures $2 B$ et $2 \mathrm{D}$; et Figure 3) ;

- les résultats d'une analyse en composantes principales sur 39 variables : effectifs de 13 CSP : ruraux recensés au lieu de résidence et au lieu de travail, urbains recensés au lieu de travail (cf. Figure 4A et 4B);

- un tableau des corrélations linéaires entre les effectifs en pourcentage pour chacune des $13 \mathrm{CSP}$, recensées respectivement au lieu de résidence dans les communes rurales, au lieu de travail dans ces mêmes communes, et au lieu de travail encore dans les communes urbaines. Ces corrélations ont été choisies par référence au modèle explicatif simple présenté dans la Figure 1. Nous avons également calculé les corrélations partielles :

- entre les effectifs résidents dans les communes rurales et les effectifs travaillant dans les communes rurales, les effectifs travaillant dans les communes urbaines étant tenus constants ;

- entre les effectifs résidents dans les communes rurales et les effectifs travaillant dans les communes urbaines, les effectifs travaillant dans les communes rurales étant tenus constants. 
Figure 3 : Combinaison des classements sur les 2 premiers axes des analyses de correspondances séparées : actifs ruraux, actifs urbains

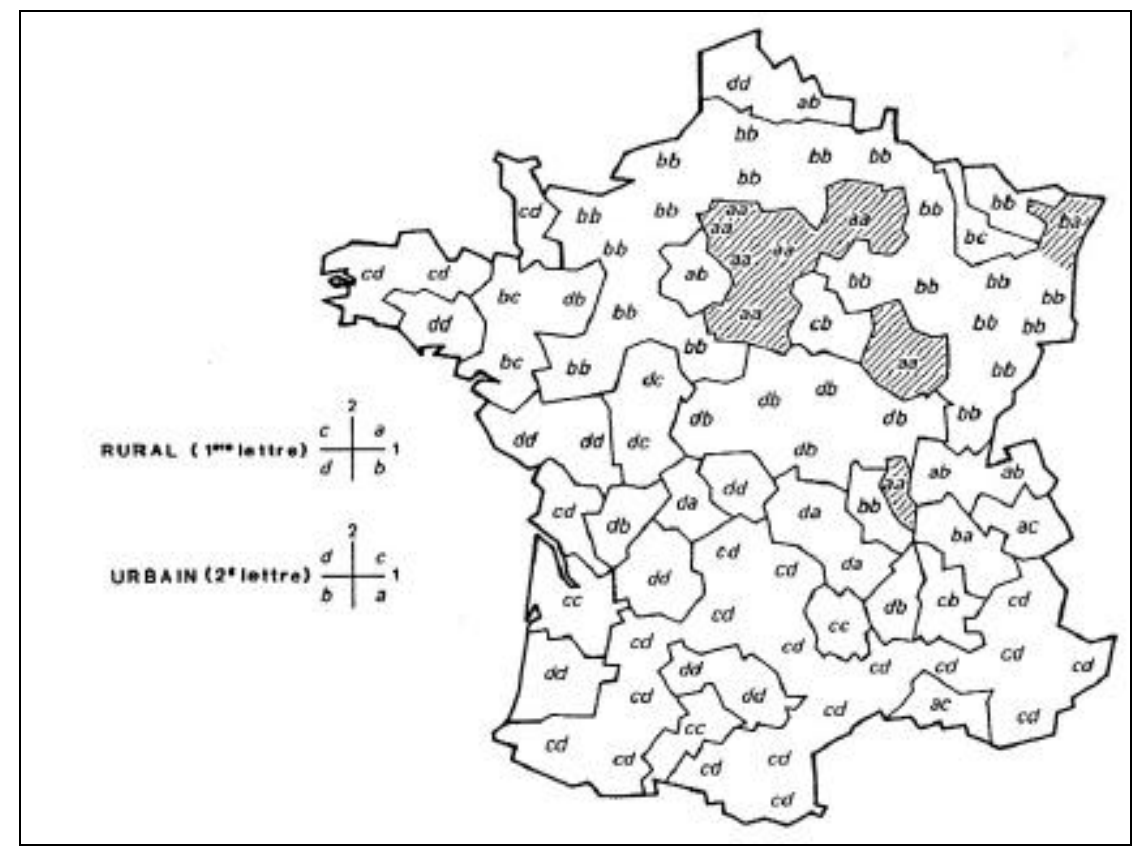

Voir texte et figures 2C et 2D pour la signification des lettres.

Figure 4A : Catégories socio-professionnelles rurales et urbaines

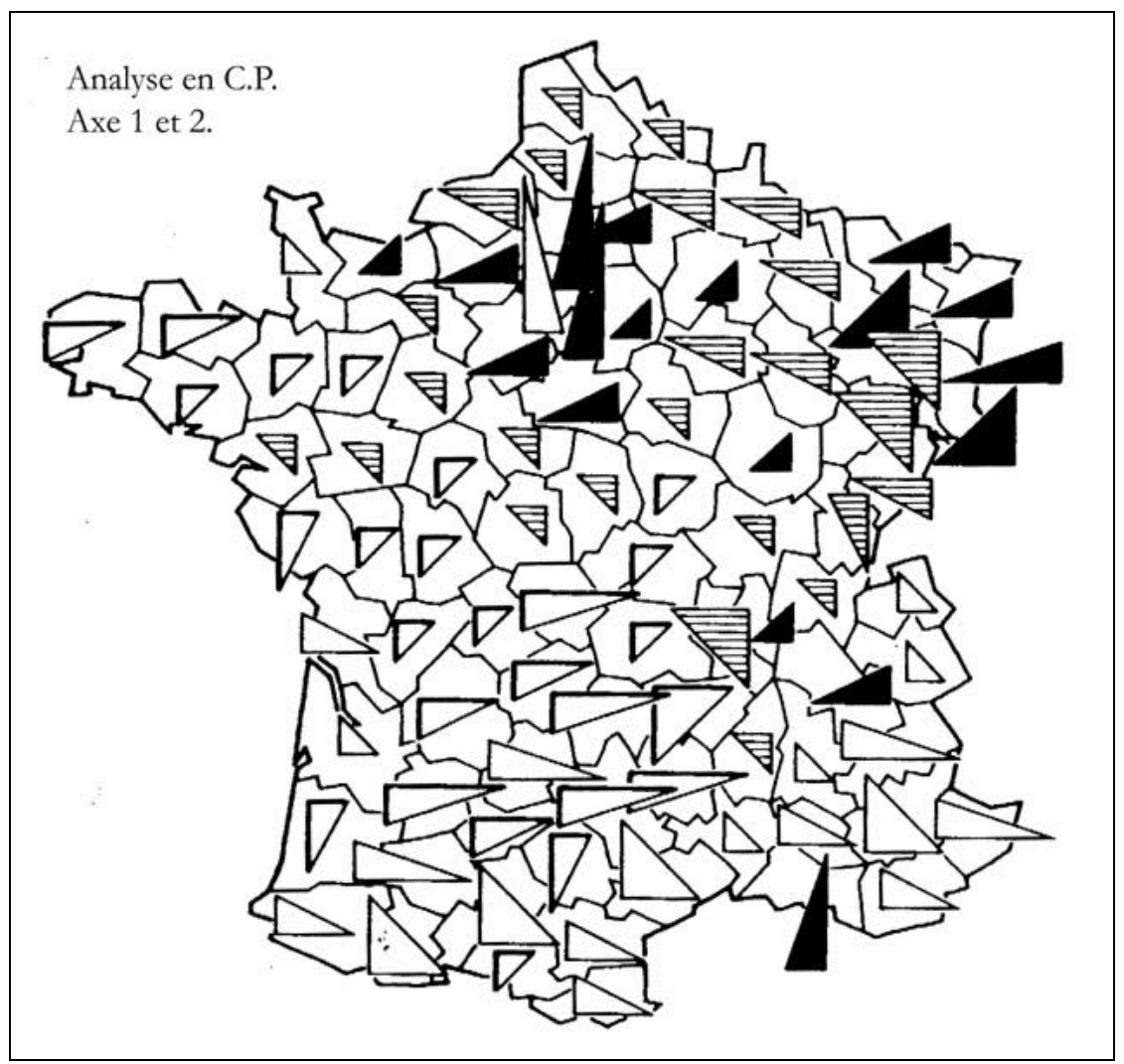


Figure 4B : Catégories socio-professionnelles rurales et urbaines

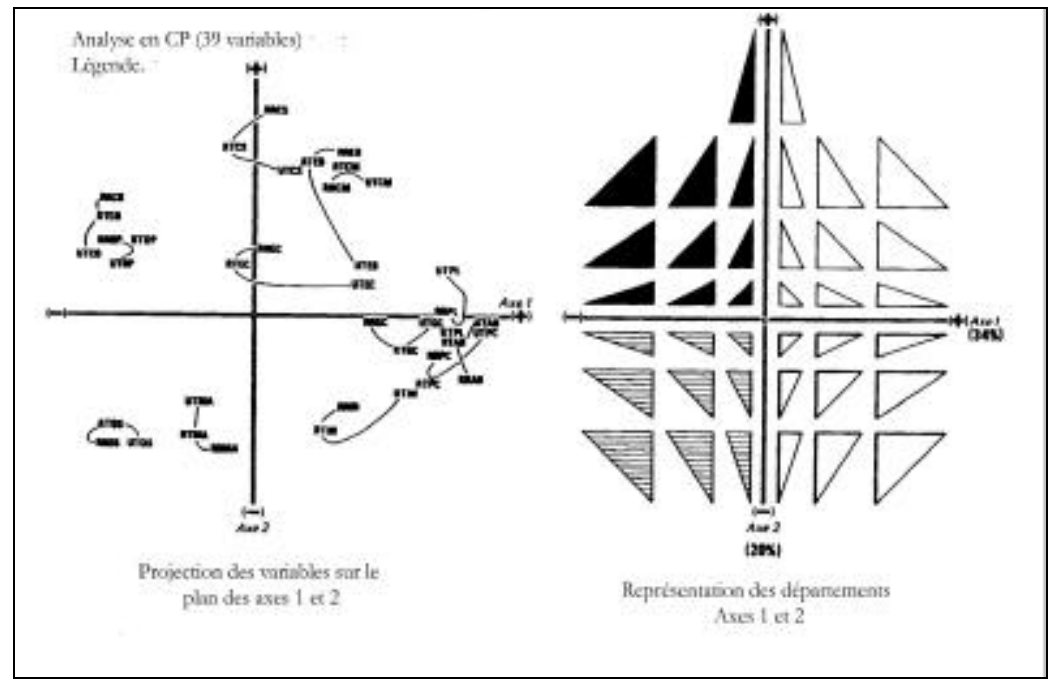

C'est surtout cette deuxième corrélation partielle qui est intéressante. Le modèle de la Figure 1 montre qu'on peut envisager une double influence des activités urbaines sur les effectifs nuraux résidents : influence indirecte, les activités urbaines et rurales étant liées, et les activités rurales déteminant largement les caractères de la population résidente ; influence directe, puisque beaucoup de ruraux vont travailler en ville. C'est cette influence directe qui est mise en évidence et mesurée par la corrélation partielle en question.

\section{L'ensemble de ces comélations est décrit par le Tableau 3.}

Tableau 3 : Corrélations entre les répartitions des CSP dans les différents espaces

\begin{tabular}{|c|c|c|c|c|c|c|c|c|c|c|}
\hline \multirow[b]{2}{*}{ CSP } & \multicolumn{3}{|c|}{ Codes } & \multicolumn{7}{|c|}{ Corrélations* } \\
\hline & $\begin{array}{c}\text { Ruraux } \\
\text { au lieu } \\
\text { de rési- } \\
\text { dence : } \\
x_{0}\end{array}$ & $\begin{array}{c}\text { Ruraux } \\
\text { au lieu } \\
\text { de } \\
\text { travail : } \\
\mathbf{x}_{1}\end{array}$ & $\begin{array}{c}\text { Urbains } \\
\text { au lieu } \\
\text { de } \\
\text { travail : } \\
\mathbf{x}_{2}\end{array}$ & r01 & r02 & r12 & $\mathbf{1 0 1 . 2}$ & $r^{2} 02.1$ & r02.1 & $\mathrm{r}^{2} 02.1$ \\
\hline Industriels & RR IN & RT IN & UT IN & 0,85 & 0,58 & 0,41 & 0,82 & 0,67 & 0,48 & 0,23 \\
\hline Petits commerçants & RR PC & RT PC & UT PC & 0,90 & 0,80 & 0,71 & 0,79 & 0,62 & 0,52 & 0,27 \\
\hline Gros commençants & RR GC & RT GC & UT GC & 0,89 & 0,64 & 0,58 & 0,82 & 0,67 & 0,33 & 0,11 \\
\hline Professions libérales & RR PL & RT PL & UT PL & 0,89 & 0,68 & 0,61 & $\mathbf{0 , 8 2}$ & 0,67 & 0,38 & $\mathbf{0 , 1 4}$ \\
\hline Cadres supérieurs & RR CS & RT CS & UT CS & 0,76 & 0,60 & 0,47 & 0,68 & 0,46 & 0,42 & 0,18 \\
\hline Cadres moyens & RR CM & RT CM & UT CM & 0,79 & 0,67 & 0,55 & 0,68 & 0,46 & 0,46 & 0,21 \\
\hline Employés de bureau & RR EB & RT EB & UT EB & 0,85 & 0,42 & 0,37 & 0,82 & 0,62 & 0,22 & 0,05 \\
\hline Employés de commence & RR EC & RT EC & UT EC & 0,58 & 0,47 & 0,18 & 0,57 & 0,32 & 0,46 & 0,21 \\
\hline Contremaîtres & RR CO & RT CO & UT CO & 0,91 & $\mathbf{0 , 8 4}$ & 0,79 & 0,74 & 0,54 & 0,48 & 0,23 \\
\hline Ouvriers professionnels & RR OP & RT OP & UT OP & $\mathbf{0 , 8 3}$ & $\mathbf{0 , 8 3}$ & 0,68 & 0,65 & 0,42 & 0,65 & 0,42 \\
\hline Ouvriers spécialisés & RR OS & RT OS & UT OS & 0,92 & 0,84 & 0,78 & 0,78 & 0,61 & 0,50 & 0,25 \\
\hline Manouvres & RR MA & RT MA & UT MA & 0,91 & 0,77 & 0,74 & 0,79 & 0,62 & 0,35 & 0,12 \\
\hline Artisans & $\mathbf{R} \mathbf{R} \mathbf{A R}$ & RT AR & UT AR & 0,94 & 0,79 & 0,69 & 0,89 & 0,79 & 0,57 & 0,32 \\
\hline
\end{tabular}

*code pour corrélation.

r01: Comélation simple entre $x 0$ et $x 1$

r02: Comélation simple entre x0 et x2

r12: Comélation simple entre $x 1$ et $x 2$

101.2 : Corrélation partielle entre $x 0$ et $\times 1, x 2$ étant constant

102.1: Comélation partielle entre $x 0$ et x2, x1 étant constant

Les coefficients de corrélation partielle élevés au carré $\left(\mathrm{r}^{2} 0 \mathrm{r}\right.$; $\mathrm{r}^{202.1)}$ donnent le pourcentage de la variation de $x_{0}$ expliqué par $x_{1}$ et $x_{2}$, respectivement, l'autre variable étant tenue constante. 


\section{Ces documents foumissent un certain nombre d'indications :}

- Les ressemblances sont nettes entre les répartitions dans les espaces urbains et les espaces nuraux, du moins quand on insiste sur les phénomènes de structure, exprimés par les données en pourcentages, sans référence aux effectifs absolus. De ce point de vue, la Figure $4 \mathrm{~B}$, qui donne les définitions des deux premiers axes de l'analyse en composantes principales sur les 39 variables, est très significative. Sur ce plan, les points représentatifs des trois modalités, nuraux au lieu de résidence (variables $R R$ désomais), nuraux au lieu de travail (RT), urbains au lieu de travail (UT) se projettent à proximité les uns des autres pour chaque CSP, sauf pour les employés, comme nous le verrons.

On retrouvera sur la carte $4 \mathrm{~A}$, à peu près les types d'espaces définis ci-dessus à partir des Figures $2 \mathrm{~A}$ et $2 \mathrm{C}$, avec cependant quelques nuances. La sur-représentation simultanée des cadres et des catégories ouvrières les plus qualifiées ( $\mathrm{OP}$ et contremaîtres) caractérise un plus grand nombre de départements que dans le cas précédent (coordonnées négatives sur l'axe 1 et positives sur l'axe 2). On retrouve bien, avec des répartitions sensiblement analogues, les espaces où dominent les tâches d'exécution, ceux où dominent les services, ceux enfin où se combinent les services et les tâches d'exécution².

- Il existe cependant des différences sensibles entre les espaces nuraux et urbains, qui sont mises en valeur par les autres documents.

La comparaison des résultats des analyses de comespondance sur les actifs résidents (cf. Figures 2, 2A-D) apporte une première série d'indications.

D'une part, les définitions des axes sont un peu différentes pour les deux analyses (cf. Figures 2C et 2D). L'opposition salariés-non salariés se retrouve dans l'analyse urbaine, mais cette fois elle conceme plus les ouvriers que les autres salariés, cadres et employés ; et surtout, cette opposition est maintenant donnée par l'axe 2, et non par l'axe 1 comme dans l'analyse rurale. De même, entre les activités qualifiées et les autres, la hiérarchie des oppositions change : elle est donnée par l'axe 1 pour l'analyse urbaine, alors qu'elle l'était sur l'axe 2 dans les communes rurales.

Malgré ces différences, on retrouve à peu près pour les villes les quatre secteurs étudiés ci-dessus dans le cas des campagnes : secteur a, avec les participants des activités de décision, cette fois mieux associés aux professions libérales; secteur $b$, encore très clairement défini par les CSP ouvrières à faible qualification ; secteur $c$, avec senvices non salariés ; mais ce secteur est défini ici avant tout par les professions libérales, beaucoup moins par les commerçants, et les employés y sont aussi sur-représentés. Le secteur d est défini par des senvices surtout commerciaux, et encore par une certaine association avec les CSP d'ouvriers peu qualifiés.

Malgré ces différences de définition, qui constituent des nuances importantes, il est possible de comparer utilement les cartes ; c'est sans doute cette comparaison qui montre les différences les plus nettes; pour la faciliter, nous avons construit la carte 3, qui indique pour chaque département le secteur dans lequel il se trouve pour l'analyse rurale (première lettre) et pour l'analyse urbaine (deuxième lettre). Il y a 16 combinaisons possibles, toutes représentées sauf une (bd). La carte 3 est donc complexe.

\footnotetext{
2 Il est intéressant de noter que les quatre types d'espaces correspondent assez bien à ceux qui ont été distingués par A. Lipietz dans ses publications sur Le capital et son espace. Correspondance d'autant plus remarquable que cet auteur porte des jugements assez sévères sur la technique de l'analyse factorielle, du moins dans ses premières publications...
} 
La moitié nord-est de la France (au sens large) est cependant caracténisée par une opposition assez simple entre des ensembles eux-mêmes simples : départements classés aa où les activités de direction et les ouvriers qualifiés sont sur-représentés; départements classés bb où sont bien mieux représentées les tâches d'exécution dans le domaine industriel ; les villes et les campagnes reçoivent le même classement dans les deux cas. Cette opposition simple caracténise une supenficie qui doit représenter entre le tiers et la moitié du pays. Il est probable qu'on puisse y voir, en suivant A. Lipietz, le domaine où la logique de la division spatiale du travail, conforme aux besoins du capitalisme contemporain, joue à plein.

Dans le reste du pays, les ensembles spatiaux sont plus petits et plus fragmentés, et les espaces nuraux et urbains reçoivent souvent des classements différents. On peut regrouper ces combinaisons en deux ensembles.

- Les services - sans activités de direction importantes - sont sur-représentés dans un premier groupe, celui des départements dont le sigle contient un c ou un d :

- combinaison cd : elle est très représentée dans le sud de la France et le nord de la Bretagne. Les actifs du tertiaire et des services sont sur-représentés dans les campagnes, les OS et manouvres y sont sous-représentés. Ils le sont moins dans les villes, qui sont par ailleurs caractérisées surtout par les fonctions commerciales ;

- combinaison cc : les campagnes ont une sur-représentation des services et une sous-représentation des $0 \mathrm{~S}$ / manœuvres, les villes ont plus de professions libérales et d'ouvriers qualifiés que dans le cas précédent. Cette combinaison caractérise la Gironde et la Haute-Garonne, et les caractères urbains sont bien évidemment dus à ceux de Bordeaux et de Toulouse ;

- combinaison dc (Vienne et Indre-et-Loire) : ce sont les campagnes qui sont relativement plus riches en $\mathrm{OS}$ et manouvres que les villes, mais les services dominent ;

- combinaison dd : dans quelques département de l'ouest (Morbihan, Vendée, Deux-Sèvres, Dordogne, les services associés à des $0 \mathrm{~S}$ et de manouvres, avec une nette sous-représentation des cadres et des ouvriers qualifiés dominent également dans les campagnes et dans les villes.

- Dans un autre groupe, la présence de la lettre b dans le signe montre le développement des tâches d'exécution :

- combinaison ba : les actifs ouvriers non-qualifiés sont sur-représentés dans les campagnes, sous-représentés au contraire dans les villes qui ont beaucoup de cadres et d'ouvriers qualifiés : ainsi, Strasbourg et Grenoble sont entourées de domaine nuraux où les tâches d'exécution sont importantes ;

- combinaison ab : dans un milieu assez industriel, beaucoup d'actifs des catégories supérieures, cadres et ouvriers qualifiés, résident et/ ou travaillent dans les campagnes (cas unique du $\mathbf{N}$ ord) ;

- combinaison bc : les services sont mal représentés dans les campagnes, qui ont des ouvriers peu qualifiés, tandis que les villes ont des services mieux développés et des industries plus complexes. Dans cette catégories sont placés des départements où une grande métropole est installée dans un ensemble régional où les tâches d'exécution industrielle sont importantes : Ille-et-Vilaine avec Rennes, Loire-Atlantique avec Nantes, Meurthe-et-Moselle avec N ancy... 
- combinaison $\mathrm{db}$ : dans le centre géométrique de la France, des villes industrielles avec beaucoup d'ouvriers non qualifiés sont entourées de campagnes où les ouvriers peu qualifiés sont moyennement sur-représentés avec des services assez importants... Dans le Puy-de-Dôme, et la Haute-Vienne, voisins de cet ensemble, les cadres supérieurs de Clemont-Ferrand et de Limoges modifient un peu le classement (combinaison da).

Cette carte (cf. Figure 3) montre bien que, sur un peu moins de deux tiers du territoire, des situations historiques compliquées ont produit des nuances importantes entre les villes et les campagnes une logique plus simple semble s'exprimer dans le reste du pays.

Il est possible de voir comment ces différences jouent selon les catégories socioprofessionnelles en se reportant au Tableau 3.

D'une manière générale, les corrélations entre les différents espaces pour les mêmes CSP sont fortes et positives, comme le faisaient attendre les résultats des analyses factorielles. Les corrélations les plus fortes sont, en général, celles qui lient les effectifs ruraux recensés au lieu de résidence et au lieu de travail : la répartition de la population active résidente dans les campagnes ressemble donc avant tout à celle de la population qui y travaille. Dans tous les cas, viennent au second rang les corrélations entre les pourcentages des CSP dans la population résidant dans les campagnes et les catégories urbaines recensées au lieu de travail ; les coefficients restent élevés et toujours largement significatifs. Les corrélations entre les pourcentages recensés au lieu de travail dans les communes rurales et urbaines ne viennent qu'au troisième rang; certains de ces coefficients sont faibles et non significatifs.

L'examen du tableau montre, mieux que les résultats des analyses factorielles, des différences significatives entre plusieurs groupes de CSP. Elles apparaissent également bien sur le graphique de la Figure 5.

Figure 5 : Classement des CSP selon 3 coefficients de comélation

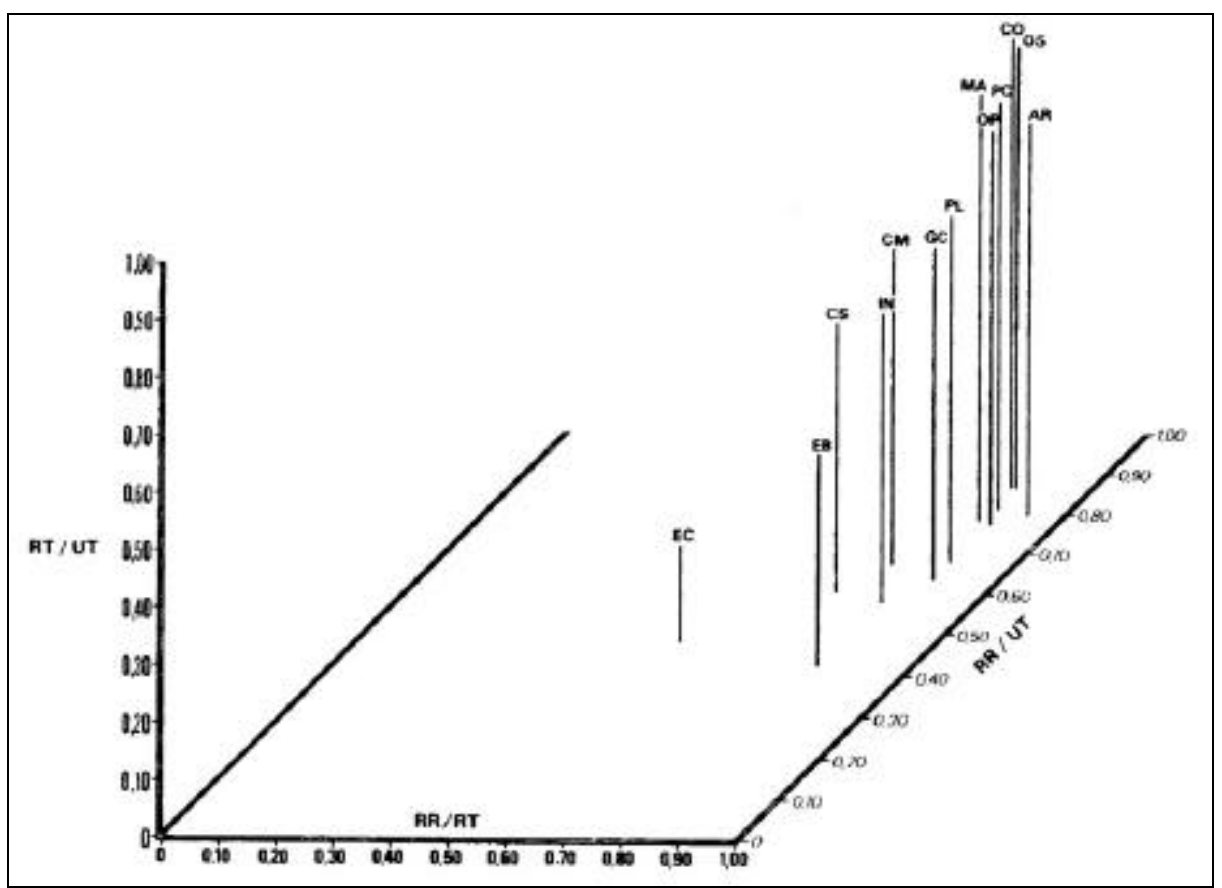


Pour six catégories socioprofessionnelles, les quatre qui concernent l'industrie (ouvriers et contremaîtres), plus les artisans et les petits commençants, toutes les corrélations sont fortes : plus de 0,90 pour la corrélation ruraux résidents/ nuraux au lieu de travail (RR/ RT), plus de 0,75 pour la corrélation ruraux résidents/ urbains au lieu de travail (RR/ UT), et plus de 0,70 pour la corrélation entre ruraux et urbains au lieu de travail (RT/ UT). Ainsi, la répartition des effectifs des ouvriers donne des pourcentages répartis de façon voisine dans les trois espaces étudiés, et il en va de même pour les artisans et les petits commerçants. Dans le cas de ces CSP jouent des régularités à l'échelle nationale, qui font sentir leurs effets dans les villes comme dans les campagnes. Les composantes qui différencient l'espace français en y créant des grandes subdivisions - les composantes régionales - l'emportent sur la composante nural/ urbain.

Dans un deuxième groupe de CSP, les corrélations sont en général plus faibles pour les professions libérales, les cadres, les industriels et les gros commerçants, les coefficients sont compris entre 0,58 et 0,68 pour la relation $R R / R T, 0,76$ et 0,89 pour la relation $\mathrm{RR} / \mathrm{RT}, 0,41$ et 0,61 pour la relation RT/ UT. Dans ce cas, des logiques explicatives différentes commencent à jouer davantage pour les répartitions dans l'espace nural et dans l'espace urbain les composantes régionales perdent de leur poids en face de l'opposition nural/ urbain.

Enfin, dans le cas des employés, surtout de commerce, les différences s'affirment entre les villes et les campagnes; pour les employés de bureau, la corrélation RR/ RT reste forte $(0,85)$, mais les deux autres sont beaucoup plus faibles (proches de 0,40 ). Pour les employés de commerce, les différences s'affirment encore, même la corrélation RR/ RT est assez basse, et celle entre RR et UT n'est pas significative.

Les corrélations partielles permettent d'affiner quelque peu la connaissance des rapports entre les répartitions urbaines et rurales. La corrélation simple entre effectifs ruraux résidents et effectifs urbains au lieu de travail (RR/ UT, ou r02 sur le Tableau 3) mesure une liaison qui s'exprime par le double circuit logique- de la Figure 1 : circuit a ou circuit direct, circuit b ou circuit indirect. Le calcul de la corrélation partielle RR/ UT, RT étant tenu constant (r02.1 sur le Tableau 3), a pour effet d'éliminer les effets du circuit b, et de mettre en évidence ceux du circuit a, c'est-à-dire du rapport direct entre effectifs des résidents nuraux et des urbains au lieu de travail, dont on peut penser qu'il résulte largement des migrations quotidiennes de travail.

Evidemment, la corrélation partielle, effet d'un seul circuit - d'un seul mécanisme - est inférieure à la corrélation simple, et cela d'autant plus que les liaisons dues au circuit dont les effets sont soustraits (le circuit b en l'occurrence), sont plus fortes. En d'autres termes, la corrélation partielle exprime l'importance du circuit a, la différence entre la corrélation simple et la corrélation partielle traduisant l'importance du circuit b.

La prise en considération de ces paramètres permet de nuancer les comportements des CSP relevés ci-dessus. 
Pour les ouvriers, les contremaîtres, les petits commerçants et les artisans, les deux circuits sont efficaces : la différence entre r02.1 est forte, mais r02.1 garde une valeur élevée, supérieure à 0,48 . Cependant, pour les ouvriers professionnels et les artisans, la mise hors jeu du circuit b a moins d'effets que pour les contremaîtres et les OS, ce qui montre que son influence est un peu moindre. L'importance du circuit a dans le cas des artisans et des petits commerçants est quelque peu étonnante, puisqu'on aurait pu penser a priori que, l'activité de ces CSP s'effectuant largement à leur lieu de résidence, les migrations campagnes-villes influenceraient assez peu leur répartition.

Dans le cas des cadres, des employés, des industriels, la corrélation partielle r02.1 est assez proche de la comélation simple r02. Le circuit b joue donc un moindre rôle dans l'explication de la corrélation d'ensemble, elle même plus faible ; l'effet direct, a, est donc plus important : la part des migrations du travail dans l'explication de la part de ces CSP parmi les résidents ruraux est forte ; ceci est particulièrement vrai pour les employés de commerce et les industriels.

Les cas des professions libérales et des gros commerçants sont intermédiaires.

Les cartes des Figures $6 \mathrm{~A}$ à $6 \mathrm{E}$ données à titre d'échantillon pour cinq $\mathrm{CSP}$, permettent évidemment d'apprécier visuellement les différences révélées par les coefficients de corrélation. L'étude de ces cartes permet aussi de faire des hypothèses sur la nature des composantes régionales et des oppositions nural/ urbain qui jouent dans les différents cas; mais il nous parait nécessaire de réserver ces commentaires pour une phase ulténieure; en effet, le jeu des composantes différentes, ou mieux, de logiques de localisations variées, est aussi bien mis en évidence par l'étude des autocorélations spatiales, et celles-ci doivent être décrites d'abord. 
Figure 6 : Part relative de 5 CSP dans 3 espaces

(ruraux résidents ; ruraux au lieu de travail ; urbains au lieu de travail)

Les cartes indiquent le pourcentage de chaque CSP dans les départements.

Les coupures ont été faites de façon à mettre en évidence la moyenne $(\mathrm{m})$; les tranches sont d'un demi écart-type. Sur les cartons-légendes, on a indiqué les valeurs centrées-réduites (VCR) et les pourcentages correspondants pour chaque variable (\%).

La valeur du coefficient «c » de Geary est indiqué à côté de chaque carte. Un graphique indique les comélations entre les répartitions représentées par chaque carte (comélations simples).

Figure 6A : Contremaîtres

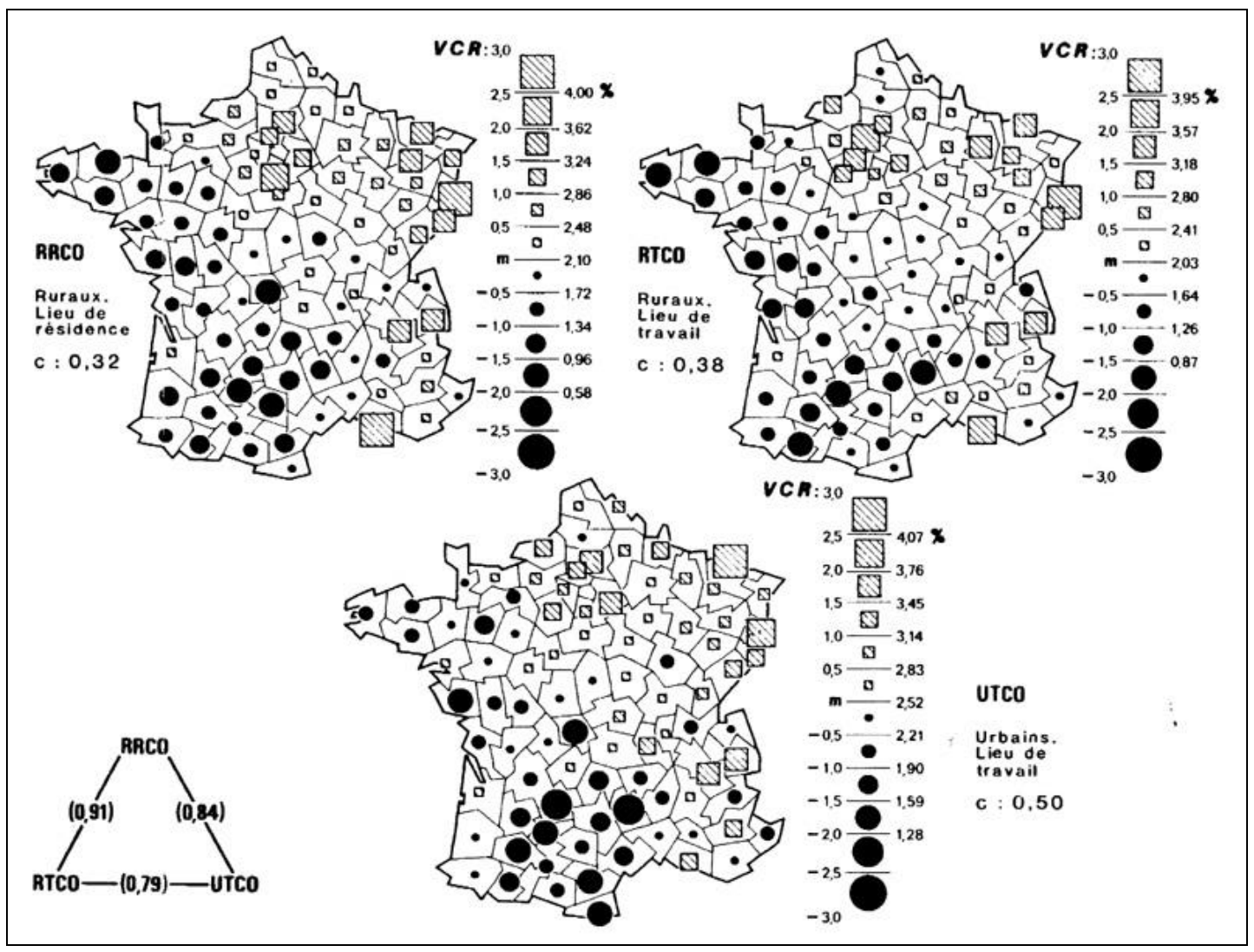


Figure 6B : Manouvres

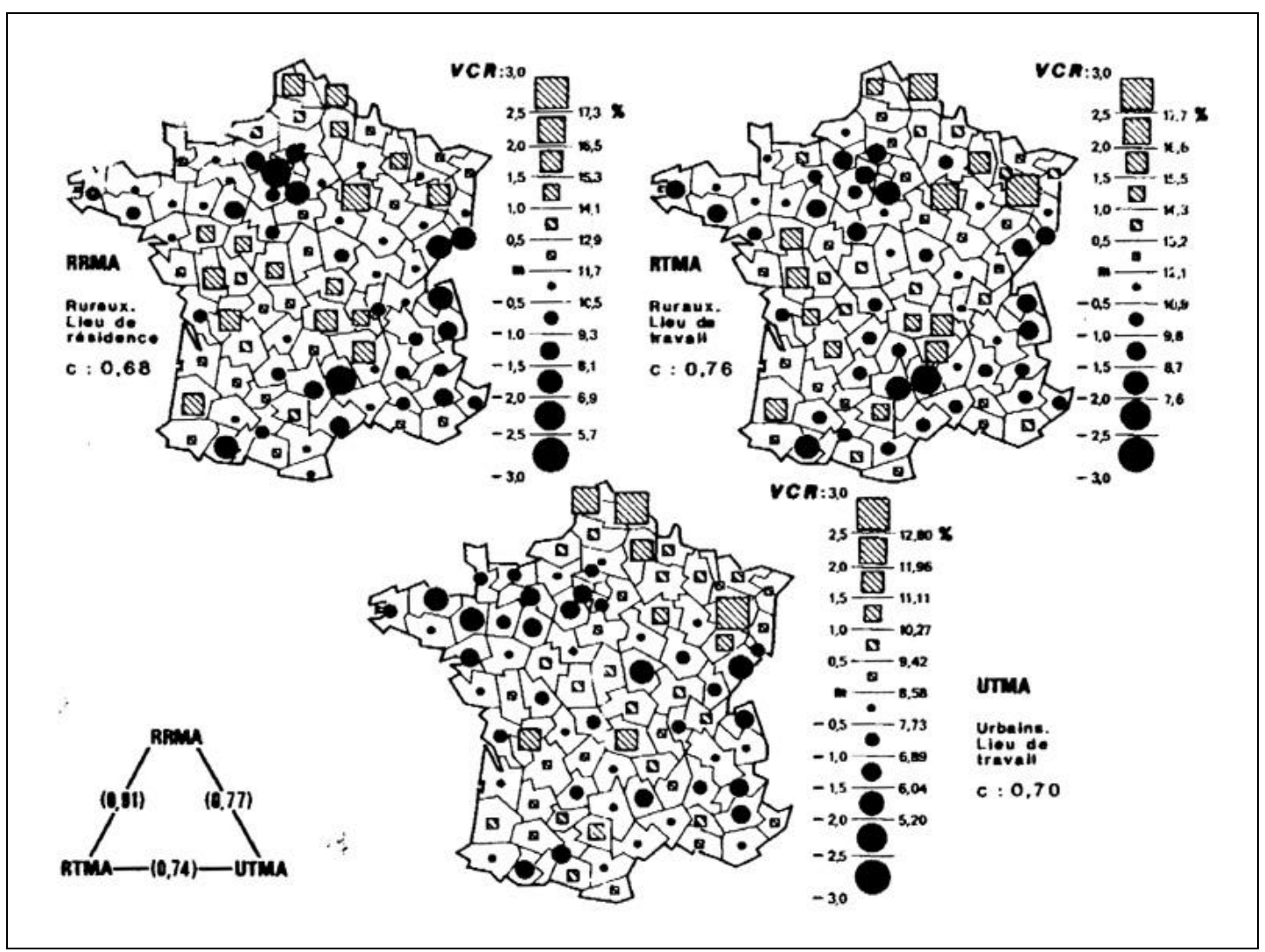

Figure 6C : Professions libérales

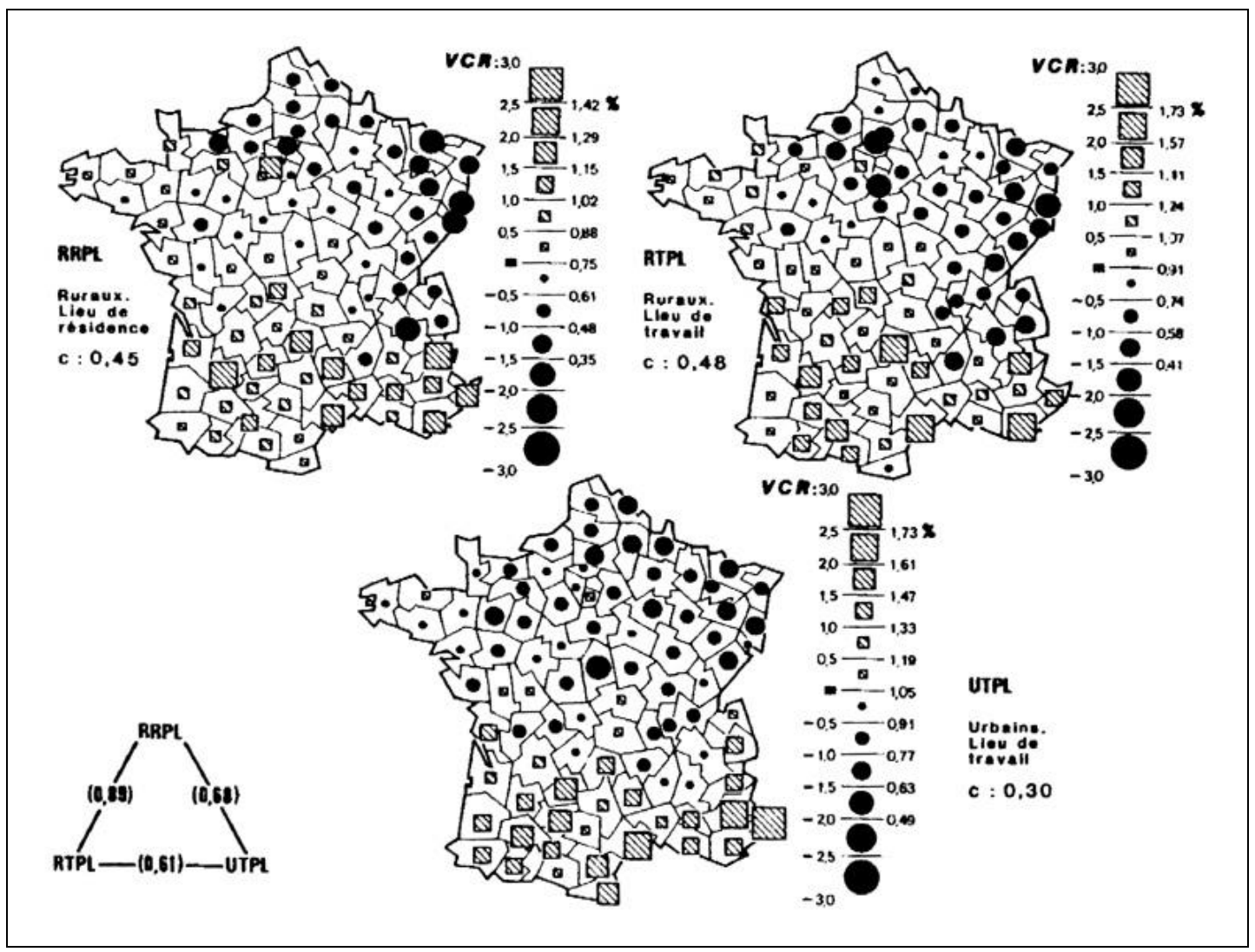


Figure 6D : E mployés de bureau

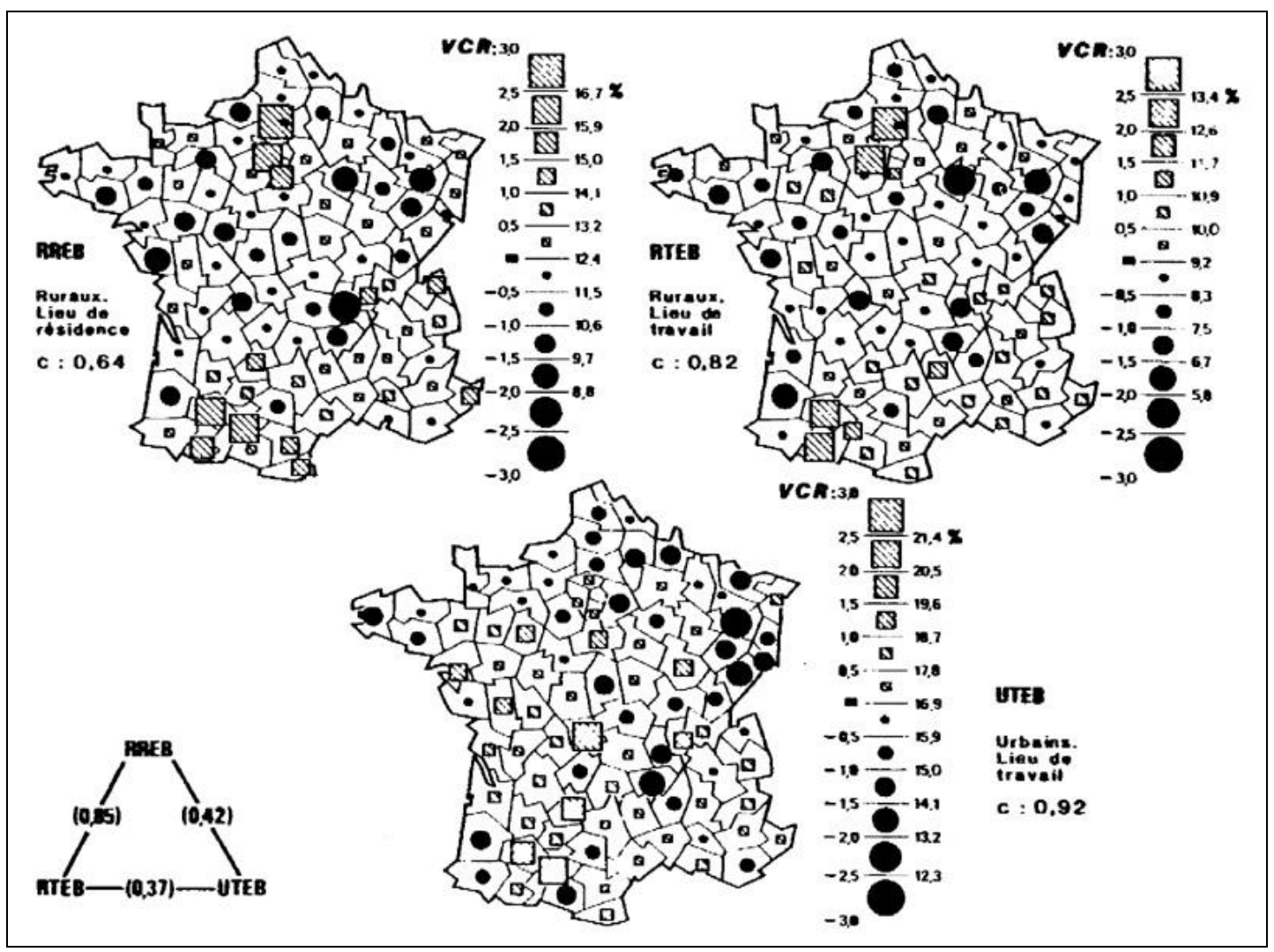

Figure 6E : Cadres supérieurs

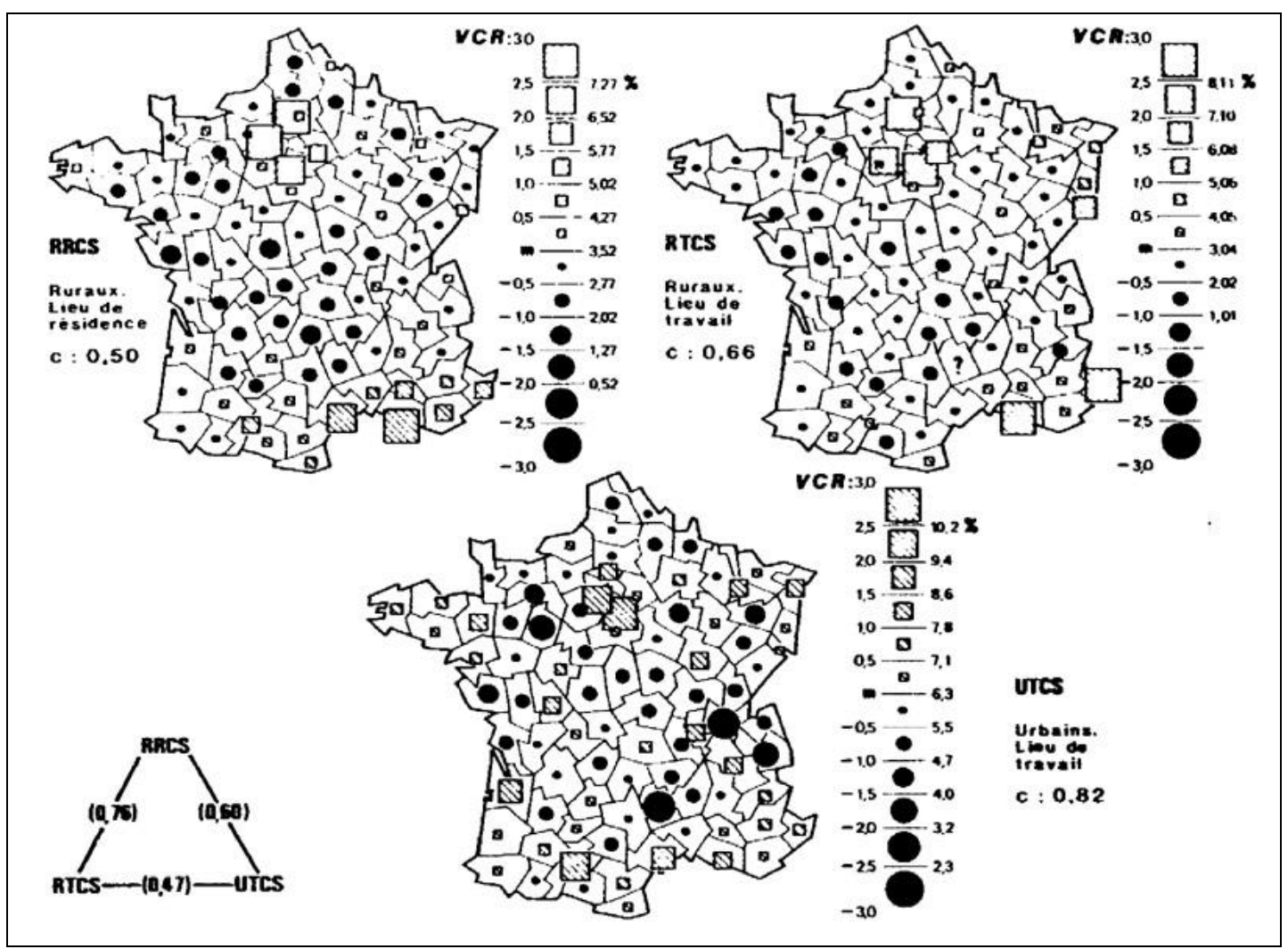




\section{Les autocomélations spatiales}

Dans un espace donné, partagé en n subdivisions, chacune de ces subdivisions étant caractérisée par une valeur de la variable étudiée, trois cas limites sont possibles :

- Les valeurs des subdivisions proches tendent à être voisines : soit qu'une valeur élevée dans une subdivision induit une valeur élevée dans les subdivisions voisines (et il en va de même pour les valeurs faibles), soit qu'une cause ou un ensemble de causes tendent à induire des valeurs élevées dans les mêmes groupes de subdivisions (et de même pour les valeurs faibles). On dira que, dans ce cas, il existe une autocomélation spatiale dans la répartition des valeurs de la variable dans les différentes subdivisions. (Il convient de remarquer que, dans le cas des autocomélations comme de toutes les corrélations, il existe deux modèles explicatifs possibles : ou bien A influence B et/ ou réciproquement, ou bien A et B subissent une influence commune qui tend à établir un lien entre les valeurs de A et de B. Il est probable que dans le cas des autocorrélations spatiales, c'est très souvent le second modèle explicatif qui est valable. Certaines réticences exprimées vis-à-vis de la notion d'autocorrélation spatiale viennent sans doute de ce qu'on a cru qu'elle ne faisait référence qu'au premier modèle explicatif. Pour éviter toute ambiguïté, il est parfois préférable de dire que les coefficients d'autocorrélation mesurent la cohésion spatiale des distributions).

- Les valeurs des subdivisions proches tendent à s'éloigner les unes des autres, une valeur élevée dans une subdivision tendant à induire des valeurs faibles dans les voisines et inversement. Dans ce cas, on a une autocorrélation faible, et une disposition de type échiquier - les cases du jeu d'échec ou de dames correspondant à une disposition de ce genre lorsque la variable n'a que deux modalités.

- $\mathrm{Si}$ aucune de ces deux actions ne joue, on obtiendra des groupements de valeurs fortes et faibles peu accentués, ressemblant à ceux que l'on pourrait obtenir en tirant au sort les valeurs à affecter à chaque subdivision ; c'est pour cela qu'on dit que de telles répartitions sont de type aléatoire.

En face d'une répartition réelle, il est utile de disposer d'un test qui permette de savoir si cette répartition s'écarte de façon significative d'une répartition aléatoire, et de comparer les degrés de cohésion (ou d'autocorrélation) existant entre les répartitions de variables différentes.

Geary a proposé un indice d'autocorrélation (indice c) dont la formule de calcul est la suivante :

où l'on $\mathbf{a}$ :

$$
c=\frac{n-l \sum \sum d_{i j}\left(x_{i}-x_{j}\right)^{2}}{4 l \sum\left(x_{i}-\bar{x}\right)^{2}}
$$

$\mathrm{X}_{j}$ et $\mathrm{X}_{i}$ : ensemble des valeurs de la variable pour les différentes subdivisions, la moyenne de la variable,

1 : nombre de contiguïtés entre subdivisions (il y en a 211 pour les départements français pris en considération ici, en comptant que les départements entourant l'agglomération parisienne sont contigus entre eux à travers les départements de Paris et de la petite couronne laissés de côté ici). 
Le numérateur du coefficient somme toutes les différences entre les départements contigus : $d_{i j}$ représente une matrice carrée où les subdivisions (ici les départements) figurent en ligne et en colonne, les cases de la matrice comportant des 1 quand les départements sont contigus et des 0 dans le cas contraire La matrice des différences des valeurs de la variable entre tous les départements est multipliée par cette matrice de contiguïté $\left(d_{i j}\right)$, ce qui revient à ne conserver que les différences entre départements contigus. Cette somme de différences est comparée à la variance de la variable (somme de $\mathrm{Xi}-\bar{X})^{2}$. Des pondérations par le nombre de subdivisions (n) et par le nombre de contiguïtés (l) sont effectuées, afin que l'indice soit égal à 1 dans le cas d'une distribution aléatoire. La répartition est d'autant plus cohérente ou autocorrélée que l'indice c est plus faible, c'est-à-dire que la somme des différences entre subdivisions voisines est faible par rapport à la variance de la variable. Geary propose un test de signification, qui permet de dire dans quelle mesure la répartition est significativement différente de la répartition aléatoire. Ce test, que nous avons effectué mais que nous ne décrirons pas ici, utilise surtout le nombre de contiguïtés et de subdivisions ; pour une même carte, la différence avec l'aléatoire est donc prouvée tant que le coefficient c est inférieur à une certaine valeur, la même pour toutes les variables. Dans le cas des départements français, cette valeur est de 0,62 pour le niveau de signification de 0,01 et de 0,73 pour le niveau de signification de 0,05 .

Le Tableau 4 donne les valeurs de l'indice c pour 36 variables (soit 12 CSP dans 3 types d'espace), les difficultés techniques nous ayant obligé de laisser de côté ici la variable « industriels ».

Le tableau montre d'abord que, dans l'ensemble, les autocorrélations spatiales sont fortes : sur les 36 cas envisagés, 8 seulement ne sont pas significativement différentes de l'aléatoire au niveau de signification de 0,05 . D'une manière générale, les répartitions dans l'espace rural sont mieux autocorrélées que celles concemant les CSP urbaines recensées au lieu de travail (cf. Tableau 4). Ceci est d'ailleurs confirmé par les résultats du test auquel ont été soumises les coordonnées des départements sur les trois premiers axes des deux analyses en correspondances séparées, portant, rappelons-le, sur les CSP recensées au lieu de résidence : les trois valeurs pour l'analyse urbaine sont décalées vers les valeurs fortes de l'indice c, (donc vers des autocorrélations plus faibles), par rapport aux valeurs pour les données nurales; le fait est particulièrement net pour l'axe 1 de l'analyse urbaine.

La valeur forte des autocorrélations n'est guère surprenante; la répartition de la population active reflète en effet les grands contrastes socio-économiques de l'espace français, qui sont créateurs d'homogénéité sur des aires étendues. Cependant, il existe des différences assez importantes entre les CSP.

D’une part, certaines CSP ont des répartitions présentant bien plus de cohésion que d'autres ; le tableau montre un contraste net entre les cohésions fortes, globalement, pour des catégories comme les artisans, les contremaîtres, les petits commerçants et les professions libérales, et des cohésions plus faibles pour les employés, les $0 \mathrm{P}$, les manouvres, les cadres moyens. D'autre part, les différences entre les cartes rurales au lieu de résidence et au lieu de travail et les cartes urbaines ont des aspects variés. La cohésion est à peu près la même pour des cartes comme celles des artisans, des manoeuvres, des OS. Elle diminue assez fortement au contraire des résidents ruraux aux travailleurs ruraux et aux travailleurs urbains pour les contremaîtres, les petits 
commerçants, les $\mathrm{OP}$ et les cadres moyens, et bien plus fortement pour les cadres supérieurs et les employés de bureau. Plus rares sont les cas où l'indice c diminue des campagnes aux villes, comme pour les gros commerçants, les professions libérales et les employés de commerce. L'étude des cartes, dont certaines sont présentées dans la Figure $6 \mathrm{~A}$ à $\mathrm{E}$, permet d'envisager des hypothèses explicatives de ces nuances. Mais comme elles sont souvent valables aussi pour les variations constatées ci-dessus à propos des corrélations, il est possible de traiter ensemble les deux problèmes.

Tableau 4 : Valeurs du coefficient de Geary ; 40 variables (coefficient c)

\begin{tabular}{|c|c|c|c|c|c|}
\hline \multirow[t]{2}{*}{ Valeurs de c } & \multirow{2}{*}{$\begin{array}{l}\text { CSP : Rurales } \\
\text { lieu de résidence }\end{array}$} & \multirow{2}{*}{$\begin{array}{l}\text { Variables: } \\
\text { CSP : Rurales } \\
\text { lieu de travail }\end{array}$} & \multirow{2}{*}{$\begin{array}{l}\text { CSP : Urbaines } \\
\text { lieu de travail }\end{array}$} & \multicolumn{2}{|c|}{$\begin{array}{l}\text { Coordonnées, } 3 \text { premiers axes, } \\
\text { analyses factorielles } \\
\text { (correspondances) }\end{array}$} \\
\hline & & & & Rurales & Unbaines \\
\hline \multirow{4}{*}{0,30} & AR & & & & \multirow{6}{*}{ RU2 } \\
\hline & Cr & & PL & RR1 & \\
\hline & 60 & & & & \\
\hline & PC & CO & & & \\
\hline \multirow{3}{*}{0,40} & & AR & $\begin{array}{l}\text { PC } \\
\text { AR }\end{array}$ & & \\
\hline & & & GC & & \\
\hline & PL & & & & \multirow{5}{*}{ RU3 } \\
\hline \multirow[t]{3}{*}{0,50} & $\begin{array}{l}\text { CS } \\
\text { OS }\end{array}$ & PC & CO & RR2 & \\
\hline & & & & & \\
\hline & oc & GC & OS & & \\
\hline \multirow[t]{4}{*}{$\mathbf{0 , 6 0}$} & & os & & RR3 & \\
\hline & OP.EB & OP & EC & & \\
\hline & CM & CS & $\mathbf{C M}$ & & \\
\hline & MA & & & & \\
\hline 0,70 & EC & & MA & & RU1 \\
\hline \multirow{4}{*}{0,80} & & MA & $\mathbf{O P}$ & & \\
\hline & & EB & & & \\
\hline & & & CS & & \\
\hline & & CIM & CM & & \\
\hline 0,90 & & EC & & & \\
\hline
\end{tabular}

Code des variables : CSP, (colonne 2 à 4$)$ : voir Tableau 1.

RR1, RR2, RR3 : coordonnées s/ axes 1, 2, 3 de l'analyse en corr. : ruraux au lieu de résidence

RU1, RU2, RU3 : coordonnées s/ axes 1, 2, 3 de l'analyse en corr. : urbains au lieu de résidence

Variables au dessus du double trait: autocomélation différente de l'aléatoire au niveau de signification de 0,01; au dessus du trait unique : autocomélation différente de l'aléatoire au niveau de signification de 0,05. 


\section{Autocorrélations, comélations et composantes explicatives}

Comme les cartes qui diffèrent par leur degré de cohérence sont aussi relativement mal comélées entre elles, il a été possible de classer les CSP du double point de vue de la cohérence et de la variété urbaine/ rurale de leurs répartitions. (cf. Tableau 5). Le commentaire de ce tableau suggère des jeux de logiques explicatives différentes.

- Pour un premier groupe de CSP, la cohésion est forte dans des répartitions qui sont très proches pour les trois catégories de variables (cas des contremaîtres, des artisans et des petits commerçants). Dans ces cas, on enregistre l'action d'une logique explicative simple, avec peu de composantes (nous appelons composante explicative un système causal ou une série causale cohérente). De plus, ces composantes peu nombreuses sont productrices d'homogénéité, dans la mesure où elles jouent sur des aires étendues, et où des valeurs élevées en un lieu ne tendent pas à produire des valeurs plus faibles à proximité. Par exemple, la répartition des contremaîtres (carte 6A) reflète assez simplement l'opposition entre la France industrielle et la France moins industrielle. La différenciation de part et d'autre de la très classique ligne Le Havre-Marseille produit évidement une image cohérente. On retrouve de la même façon une opposition simple, $\mathrm{N}$ ord-Sud cette fois, dans le cas des petits commerçants et des artisans. (Les causes qui jouent pour expliquer ces contrastes sont nombreuses et complexes, on le sait, mais on peut considérer qu'elles se regroupent en une composante unique).

- Dans un deuxième groupe de CSP, les corrélations sont fortes entre les variables rurales et urbaines, mais la cohésion des répartitions est plus faible. C'est que la logique explicative est plus complexe que dans le cas précédent, tout en jouant de la même façon dans les différents espaces. Par exemple, pour les manouvres, deux composantes entrent en interaction : le niveau général d'industrialisation, bien sûr, mais aussi la nature des fabrications, et même, ce qui serait une troisième composante, la combativité de la classe ouvrière qui réussit à obtenir que soit classés comme oS des ouvriers qui effectuent des opérations qui leur valent dans d'autres branches ou dans d'autres régions, d'être classés comme manouvres. Ainsi, les manouvres sont-ils bien plus nombreux dans le textile que dans, par exemple, la métallurgie. Le cumul de ces effets a pour conséquence une multiplication des régions où les manœuvres sont sur-représentés ; elles sont nombreuses et dispersées, ce qui diminue la cohésion de la carte, mais ce sont les mêmes dans les trois espaces, d'où les corrélations fortes. Il en va de même, dans des conditions un peu différentes, pour les OS et les OP (dans le cas de ces derniers, on note des pourcentages élevés dans les départements dispersés où il y a des chantiers navals et des arsenaux d'état, ce qui diminue la cohésion de la carte).

- Les corrélations sont moyennes et les cohésions différentes dans les espaces ruraux et urbains, quand interviennent des composantes complexes, jouant différemment dans les villes et les campagnes. Dans le cas des professions libérales (carte 6C), la cohésion est plus forte pour les villes que pour les campagnes. Pour les premières, on observe une opposition Nord-Sud assez simple ; pour les secondes, cette opposition se retrouve, mais il y a également des pourcentages assez élevés dans un certain nombre de départements de l'ouest, pour des raisons qui ne sont pas évidentes, et une forte diffusion des membres des professions libérales dans les communes rurales des départements proches de Paris, ce qui augmente les gradients de variation autour de cet ensemble. Le cas des gros commerçants est comparable. Ici, la moindre cohésion de la carte rurale est liée à une sur-représentation dans les départements littoraux, moins nette dans les villes. Cet alignement de départements à valeurs fortes, 
contrastant avec ceux de l'intérieur, notamment le long de l'Atlantique, augmente la valeur du coefficient c.

Les cadres supérieurs nous offrent un exemple du cas, plus fréquent, où les cartes rurales sont plus cohérentes que la carte urbaine (cf. Figure 6D). Celle-ci montre surtout des sur-représentations dans les départements où se trouve une grande ville ayant des fonctions métropolitaines. 0 r ces villes ne peuvent être proches les unes des autres, et sont disposées selon une logique ponctuelle depuis longtemps mise en évidence dans le modèle des lieux centraux, qui gouveme, par exemple, l'industrie ${ }^{3}$. Pour des raisons qui ne sont pas évidentes, ces concentrations de cadres dans les métropoles ont souvent été sans effet très notable dans les campagnes environnantes, si bien que les cartes nurales sont bien plus simples, enregistrant seulement le contraste majeur entre la région parisienne et le reste de la France, et l'originalité du Midi méditerranéen. Des mécanismes assez analogues jouent dans le cas des cadres moyens.

- Dans un dernier groupe de CSP, essentiellement les employés, des logiques différentes produisent dans les campagnes et dans les villes des répartitions différentes, et peu cohérentes. La répartition des employés de bureau dans les communes urbaines obéit à une logique à composantes multiples, dont certaines tendent à créer des répartitions ponctuelles. En effet, cette CSP est représentée fortement dans les départements à grandes villes à fonctions métropolitaines, - ce qui nous renvoie au cas des cadres supénieurs - mais aussi - autre composante - dans les départements peu urbanisés, où une ville, souvent le chef-lieu, a des fonctions administratives dominantes. Les fortes valeurs se retrouvent donc dans des départements nombreux, dispersés, inégalement répartis. Dans les espaces ruraux, cette deuxième composante ne se fait plus guère sentir, et la carte est plus simple et très différente : elle enregistre seulement la part relative forte des employés de bureau dans le sud et la région parisienne.

Tableau 5 : Caractères de la répartition des différentes CSP

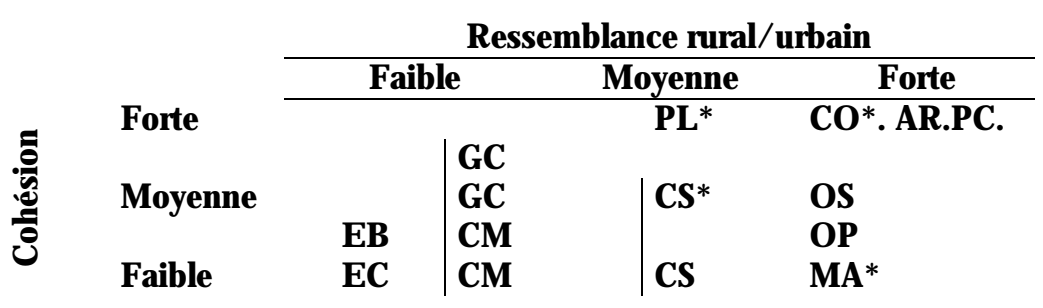

NB. Voir Tableau 1 pour le code des variables.

Le sigle a été répété pour les CSP dont la répartition présente des cohésions nettement différentes dans les 3 espaces considérés.

* Répartitions cartographiées sur la Figure 6.

Ces exemples montrent assez bien que les variations des coefficients de corrélation et d'autocorrélation dépendent à la fois de la nature des causes en jeu - plus ou moins créatrices de répartitions homogènes ou ponctuelles - et du nombre de composantes explicatives.

\footnotetext{
${ }^{3}$ L'importance des économies d'agglomération dans la localisation industrielle explique qu'elle puisse être fortement représentée dans l'ensemble d'un département et dans des ensembles de départements voisins. D'autre part, les activités de transformation se diffusent davantage dans les petites villes et les campagnes que les activités de direction et les services supérieurs.
} 
Cette étude a porté sur des phénomènes où les corrélations et les autocorrélations sont globalement élevées; elle nous a donc amené à étudier des nuances plus que de grands contrastes. Mais elle nous a cependant montré, croyons-nous, comment des calculs sur les répartitions mettent assez bien en évidence des problèmes de logique explicative, et comment des considérations passablement géométriques conduisent à préciser le jeu de forces économiques et sociales - peut-être en définitive les structures spatiales résultant d'un mode de production. Il nous semble donc que ces calculs se trouvent assez largement justifiés, et que, en particulier, l'étude d'indices comme celui de Geary vient très utilement compléter et préciser l'étude visuelle des cartes.

Travaux de l'Institut de Géographie de Reims, 1981, nº 47, p. 39-47. 